\title{
A Survey on Advanced Approaches of EHR in inter-related data using Machine Learning
}

\author{
T.V.M. Sairam, R. Rajalakshmi \\ School of Computing Science and Engineering, \\ Vellore Institute of Technology, Chennai, India \\ venkata.manikanta2016@vitstudent.ac.in,rajalakshmi.r@vit.ac.in
}

\begin{abstract}
Medical data is being used for huge number of research works over the globe which is for predicting something novel case studies in each work. The current research which we are handling is on utilizing the EHR (Electronic health Records) data in an efficient way based on the cause - effect ratio and the variables available for the data manipulation, processing and generating efficient data for designing efficient prediction models. In this research we are focusing on the congenital tethered cord syndrome through which some many functional outcomes issues are recording in different cases and there is a wide range of scope for research. In this research we are identifying the data from different EHR applications and designing the architecture to gather valuable data set from those for designing prediction model for predicting functional outcomes of health and life in patients with congenital deformity. Through EHR applications we gather information and BigData is being created in this sector. Data inter -relation is explained in this survey article in an efficient way with respect to medical domain. EHR data will be hosted over the cloud and in public repositories. Will focus on those categories in an efficient manner.
\end{abstract}

Index Terms-Machine Learning, Bigdata, Cloud Computing, EHR, Inter-related disease, Predictions

\section{INTRODUCTION}

$\mathrm{H}$ EALTH information system often called as electronic health records are ain focus of IT industry to develop their market in health care domain as there are several researches are going on in different places on medical concepts like Parkinson's Disease using Machine learning [1], which deals with the combination of the genetic and environmental disorders which plays a major role in this kind of diseases. Here the main question is how the way EHR is related to this kind of disease treatment and how we can do the research in this kind of theories which have clueless information because there is no major readings for findings are there for the identification of Parkinson's disorder. There is a case for adoption of Health records for the multiple theoretical perspectives of the operations in different health operations and in different cases in health operations [2]. The main criteria here is to utilization of EHR data for identifying a cause or an effect with related to the previous details of patient like treatment, medication, lab, scans etc. Every single information like job details, environment habits etc will be considered as the variables for the model design and implementation. EHR is the best practice f gathering data from the health pracctioners from different organizations. Consider a scalable mHealth application which a well known EHR which tracks all your health information and store it as sheet and we can use that as medical report and which will be directly connected to the doctors. [3]. Prediction models can be designed with this EHR data like using K-Means clustering, random forest, Decision Trees etc which will help to identify what are the variables to be considered from EHR and how we can change the architecture of EHR application[4]. Multiple Linear Regression model consists of the concept of backward elimination using which we can decide which variable of the field can be utilized for designing the model and which can be excluded. As we reduce the complexity in EHR data the best output can be achieved in the model. The variations in the functional outcome of life [5] is required the best outfit of the variables which can be gathered from EHR and apply the tools like regressions to redesign the EHR architecture and the data. The main purpose of this article is to satisfy the theory of identification of functional outcome of life in the patient with tethered cord syndrome for that case we need adaptive EHR model which will intake efficient variables and form data sets for further processing in analytics. The later sections in this paper discusses about few EHR applications in real time and how we used to get the data from those section 3 explains the requirement of cause - effect ratio and data inter-relation in section 4 we conclude this survey

\section{DiffEREnT EHR POSSIBILITIES}

\subsection{Mobile EHR}

This is a small mobile EHR application architecture which is used to guide the person or the patient 
according to his/her habits, age, weight, gender. Etc. This one is very basic architecture in EHR. Application is already programmed with all the required things and some group of combinations. Based on the input from the patient or the person it will calculate all the things and will give the result.

List of the things which that application will take are as follows. Age, Gender, Habits, Type of Job (Because of some diseases are because of the nature of work and the work location they are working etc). Algorithm will be designed and internally all the values from the patient will be taken as inputs and decode them by correlating them internally and will give a sample result. For example, it may ask to drink some amount of water frequently for time to time and ask you take some food after some time. For a short period of interval, it will give some notifications. There are some advantages as well as big disadvantage with this kind of EHR architecture. In this section, we will discuss with the architecture of this EHR system and in later section we can look forward for pros and cons.

In figure 1 we have the basic architecture of EHR application which is a mobile application using which all the patients will get the regular notifications based on the information they gave to the application.

Figure 2 describes the flow of the EHR method to take information, update in the application and do some predictions models inside and show the notifications to the user through application time to time.

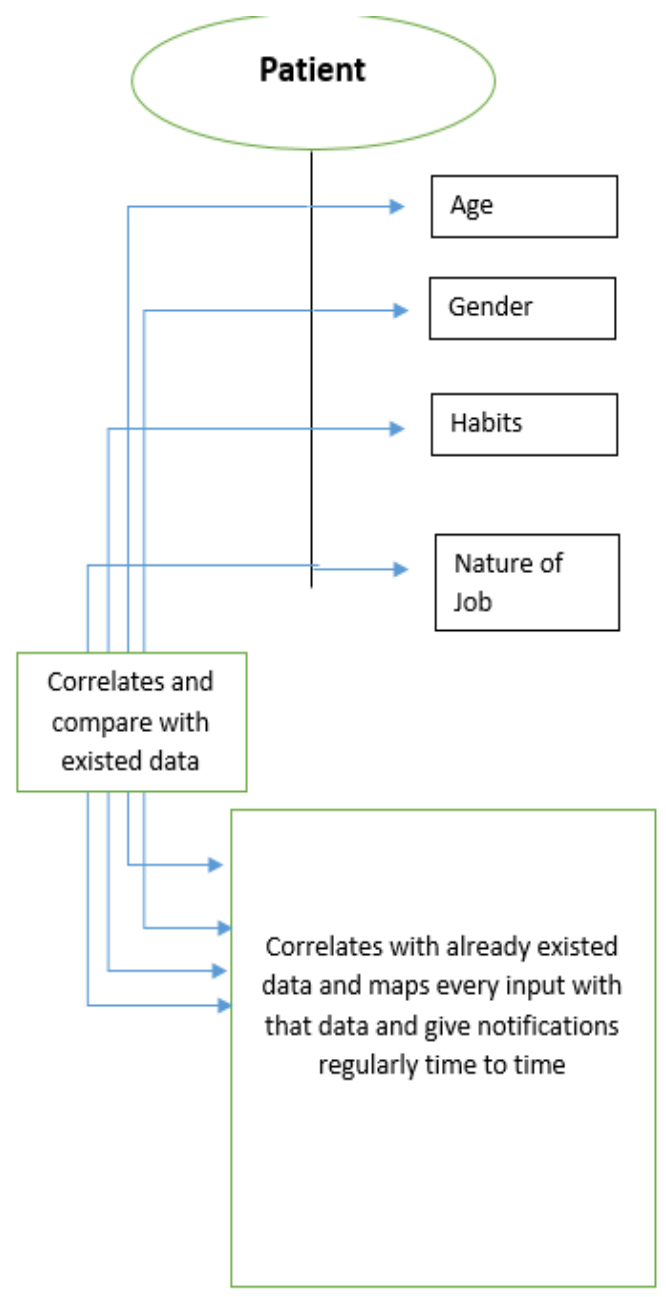

Fig 1: Mobile EHR Architecture

This comes under the unsupervised learning. If we consider machine learning in EHR it will be much useful for the users.

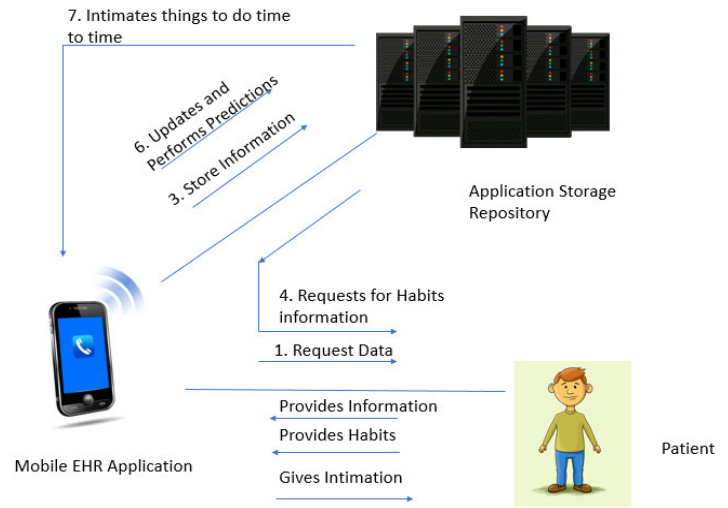

Fig 2: Data flow in Mobile EHR 


\subsection{Community Health care monitoring system}

Community Health care providers (C- HCP) are health care management system organizers which will have some man power who will come to our presence that means to our home to take all the required information about our health like blood sugar, heartbeat, oxygen levels etc. If we consider this community health care system, they have an application using which our health information can be updated and saved in global server for the purpose for further research for scholars. We can update or enter our information from any mobile application which is having the community health care management system application. Using the sensors in our mobile, all the medical information will be updates and synced simultaneously. The common feature of these kind of applications is measuring oxygen saturation using sensors. Consider Community Health care providers in the case of pregnant ladies. We need to track all the information regarding that lady, start from the BP, Sugar, Oxygen saturation level and remaining which are most important. C-HCP will come to our premises and take all the information. In low and middle income countries, we find most of the cases that pregnant ladies are losing the pregnancy because of the lack of proper health care management and treatment and also because of the late response for treatment. Economically backward families can't effort for high paid treatments, this is also one of the reason for cases of pre-eclampsia and also eclampsia period problems. In that period doctors need to check the patient continuously physically or using the mobile application.[21] Based on the information provided and stored in the server doctors need to take all the precautions to solve the problem in prior.

There are few more things to be learn about preeclampsia period ladies and their conditions. This application consists of previously successful features which are no way related to each other. 1) Predicting the situation of the patient based on identifying the risk factor mini pre-eclampsia integrated estimate risk score ( miniPIERS)[20] 2) A mobile application in phone oximeter and a sensor for pulse detection [19]. These applications predict the risks in the pregnant ladies and suggest medication and treatment for those kind of ladies in prior. The process of predicting the risk factor based on clinical validations, blood oxygen saturation
(SpO2) and also based on other common symptoms will occur in that pregnancy period. PIERS on Move ( POTM) is a low cost application for risk identification in the case of pregnant ladies. Community based implementations of cluster based randomized control of pre - eclampsia trail was discussed[22]

[4]. There are several updates are given to the original POTM application. The basic POTM application was designed to train nurse in South Africa and also some house wives who are economically backward to get treatment. So that at the time of first update for POTM nearly 202 women are assessed with the POTM application. The purpose of that trail version to know whether the POTM is operating as per the requirement or not and it is satisfying the requirements or not. So that late for the same study POTM is used by nearly 500 women with the same purpose to know the low or high risk in the pregnant ladies in pre-eclampsia and eclampsia period.

The other kind of POTM is CLIP trail. This application for community based health care providers ( C-HCP's) who are been to the women who are pregnant and test them physically or using the phone oximeter and perform some of the operations related to the pregnancy. They tested this on 500 women by approaching them personally. At each stage if their research practitioners have to give their feedback regarding their study using this CLIP trail POTM application. Prototypes are designed for the feedback for the practitioners and also to perform some of the analysis on the women or on the group of women who are pregnant. Figure 3 describes the prototype for POTM.

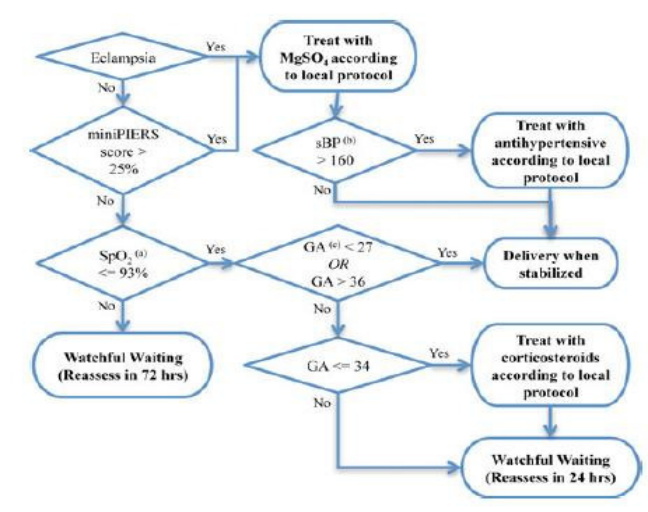

Fig 3: Diagram to know whether pregnant lady is in labor or not using POTM application. 


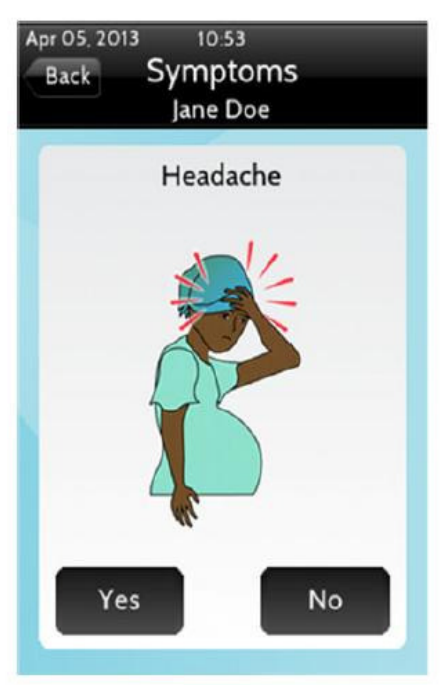

Fig 4: CLIP POTM symptom page

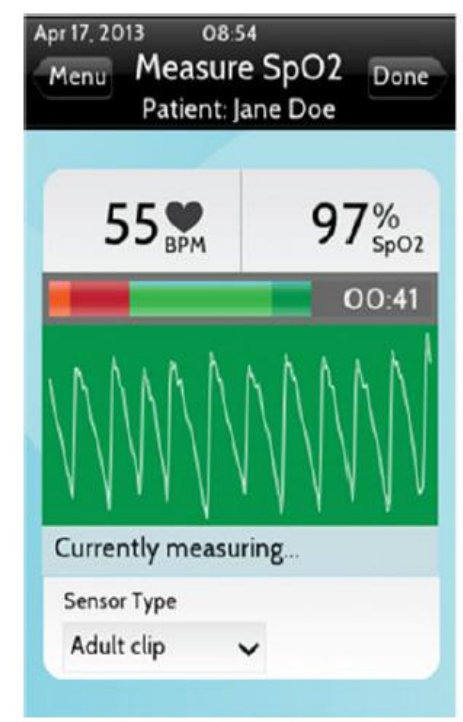

Fig 5: Oxygen saturation checker for POTM

Figure 4 explains the user interface of the application (POTM and CLIP POTM). Considering the symptoms and condition application will predict some information and will load all the required medication on the screen. Whenever oximeter recognized anything wrong in patient body it will send the information to the doctor or it may prescribe some medication meanwhile the comes and attend the case. Figure 5 indicates the oxygen saturation checker for POTM using which

\subsection{ResScan- EHR for cardiac and paralyzed patients.}

This approach is PC based personal health records management system using which doctors can analyze patient condition, update the treatment information, download the all the information stored in the device[18]. Basically, ResScan is a PC based software in which patient treatment can be simplified and this will connect to doctor's PC and he can track his patient's health condition time to time. We have an alarm in this application. Whenever patient is suffering with any problem this application will notify that to the doctor immediately. Let's see that architecture in lateral part. Initially there will be mask that patient need to wear while sleeping. In that machine (IoT) which is pre-programmed with some small embedded machine learning technology. ML included in this because whenever patient is suffering with anything and if the system recognised so, then immediately it will notify to the doctor without any third-party interaction. The readings of the patient will automatically recognised and stored in SD Card. It will show all the data it recognised in user dashboard on our PC when we log in with our credentials.

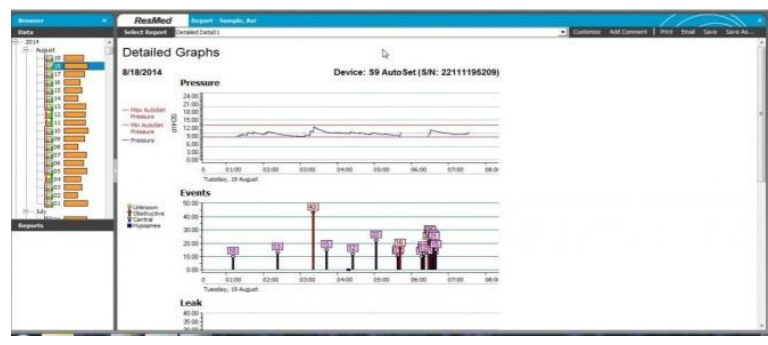
condition

Fig 6: ResScan Detailed Graph of patient

Figure 6 explains the ResScan graph format. Everything stored in the using the application will be arranged in the format of dataset. Based on the dataset graphs will created automatically and that will be delivered to the specific department in hospital or specific personal doctor of that patient.

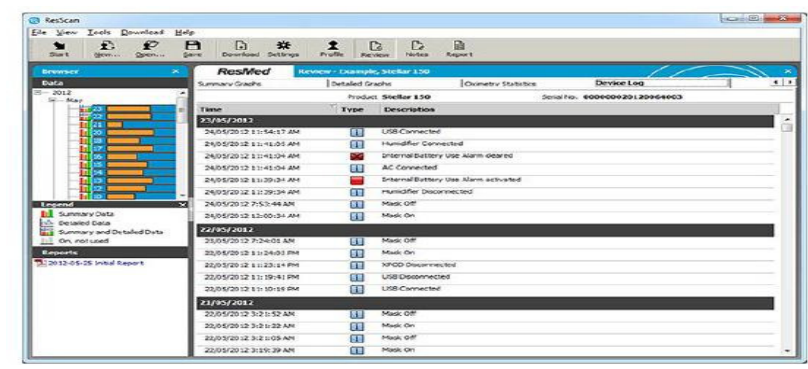

Fig 7: Device Log Tab 
Device tabs are recorded by device and stored in the internal memory of the device. Whenever we need those records, we can copy those log records and store in USB device or in any external storage devices. There are three categories of these device log tabs. 1) Alarm Events used to store information regarding the emergency intimation details that means when patient is suffering from any problem it will give an alarm to the doctor. So, that information will be given stored in the log files. 2) System events. That means information recorded by the device time to time. So that in log tabs time will be mentioned and at what time readings are taken and transferred to the doctor.

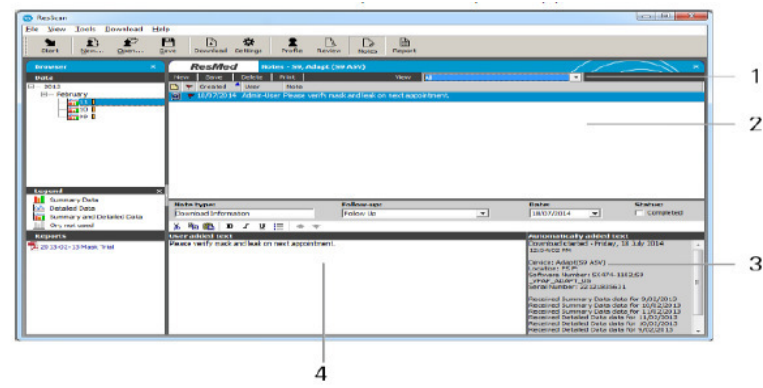

Fig 8: Device Notes Screen

In notes screen we can find the downloads done by the patient or doctor regarding the patient data. In this doctor, can store the treatment given to that patient and can review at any time he wants. Patients can create their own notes and device will create a notes screen automatically for user. In this above screen 1) Notes Toolbar 2) Note Summary 3) Automatically added text 4) Used added text.

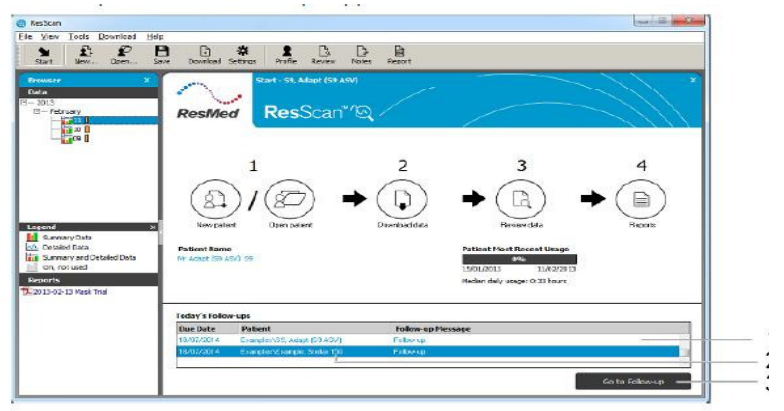

Fig 9: Follow Up page

In this follow up screen all the dues and over dues will be displayed. In the home screen go to follow up button is displayed we can directly go to that screen.

In this above screen 1) Follow ups display 2) Double click to follow up screen 3) Click button to go to follow up.

\subsection{Some common EHR models.}

The basic concept of EHR is common in all areas. Some of the common things are as follows in this block.

1) EHR in emergency department of hospital. In causality of hospital patients may join with some chronic disease or problems. So, that using this EHR concept all the patient treatment details will be recorded and start from join date everything will be stored like operation details, medication, physiotherapy etc. and all the review details, if there are any consultants from other hospital doctors etc are stored here. Based on the patient condition to give better treatment we can create graphs for better understanding of the patient condition like ResScan in previous concept.[7,8,9,10]

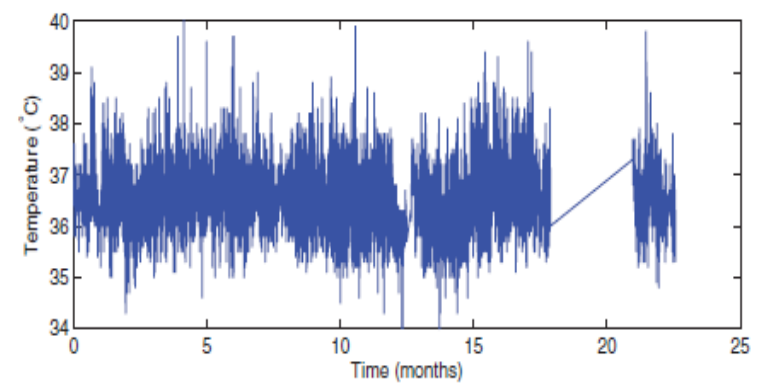

Fig 10: Time Series of Temperatures.

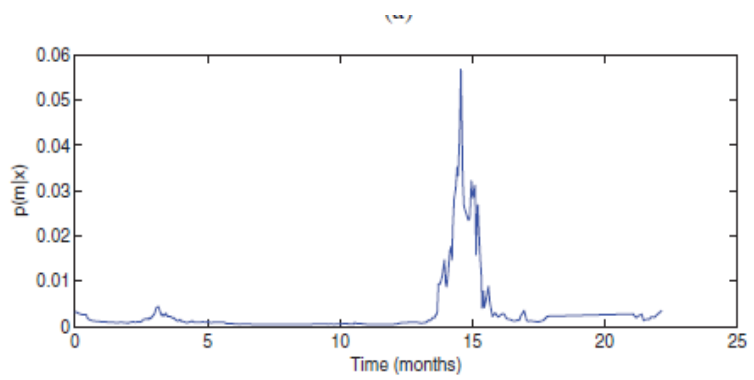

Fig 11: Bayesian Change point detector

Early warning scores are explained in[11,12,13] using which we can give scoring for the patient passed on the readings using the EHR. Based on this large dataset are maintained and monitored and graphs are designed for further process.[14,15,16,17]

2) Patient centred EHR for hospitals using which we can access our data from anywhere in this universe and we can take suggestions from any doctor by showing him the previous history of his treatment. 
Health information Technology for economic and clinical health act (HITECH) is the base for this patient centred application of EHR. Table 1 adopts the list of physician's approach regarding EHR.

Table 1: Physician's adaptation of EHR

\begin{tabular}{|c|c|c|}
\hline Category & Description & $\begin{array}{l}\text { Occurr } \\
\text { ences }\end{array}$ \\
\hline Work & $\begin{array}{l}\text { The physician perspective of } \\
\text { EHR usage on physician work. } \\
\text { Subcategories: Work Impact, } \\
\text { Productivity, Integrated } \\
\text { Collaboration, } \\
\text { Access/Connectivity, Requested } \\
\text { Enhancements, EHR Here to } \\
\text { Stay. }\end{array}$ & 102 \\
\hline Technological & $\begin{array}{l}\text { The physician perspective on } \\
\text { implications of IT Context on } \\
\text { EHR usage. Sub-categories: } \\
\text { Systems } \\
\text { Hardware \& Covelopment, } \\
\text { Physician Communication \& } \\
\text { Collaboration. }\end{array}$ & 70 \\
\hline Social & $\begin{array}{l}\text { The physician perspective on } \\
\text { implications of social context on } \\
\text { EHR usage. Sub-categories: } \\
\text { Standard Templates, Processes } \\
\text { \& Rules, Data, Interfaces \& } \\
\text { Presentation, Knowledge \& } \\
\text { Learning. }\end{array}$ & 34 \\
\hline Total & & 206 \\
\hline
\end{tabular}

Figure 12 describes the workflow of the physicians approach for the patient centred care.

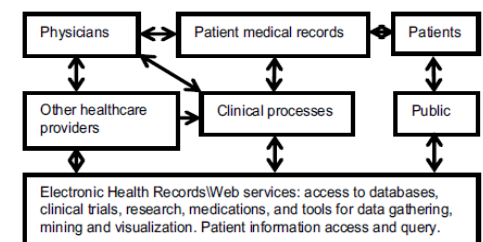

Fig12: Model for physical collaboration of patient centred care.

Many approaches are there for EHR application but there are lot of backlogs in every architecture like not able to predict that the data obtained in mHealth application is genuine or not, prediction algorithm is not using for maintaining consistency in patient self immunity capacity. Because of using anti - biotics heavily patient may lose his/her self healing capacity. A new prediction model and algorithm is introducing in this paper with a basic architecture with an example of work flow of the algorithm.

\section{Discussion}

Data Inter relation is the background architecture of EHR applications [6] which deals with identification of the common points between the variables and we can replace the entire set of the common variables in the data set with less $\mathrm{P}$ value than SL value. We can identify the variable with less $\mathrm{P}$ value using backward elimination process in the regression model. Hypothesis is drawn and we will use the entire set of EHR of data as dataset and will reduce each variable which has the highest $P$ value than SL and finally we will get the appropriate variable which suits best fit the model. Cause - Effect ratio is other thing in Machine Learning models details which predicts the effect based on the ratio of the cause set and identifies the most valuable set of causes in the available set of variables causes set. The model have to predict the cause have highest chances of causing some specific effect. There is a sample architecture must be followed with respect to the EHR data Separation and identifying the most valuable variable must be best fit in the model.

\section{Conclusion}

This survey paper demonstrates the different EHR applications and the working purpose of those in their respective areas like cardiac like ResScan, mHealth applications and the main reason for better utilization of EHR data is to provide valuable health services to the people who are economically backward. In further to this research we are focusing on designing prediction model with data mining and machine learning techniques to predict outcomes of life for the patients with congenital diseases. As we know diseases are divided into congenital and genetic, tethered cord syndrome is focused main the task to gather, clean and design related models with related to expected result. Further research carries all the benefit of utilization of the EHR models discussed in this article in an efficient manner.

\section{REFERENCES}

[1] "An improved approach for prediction of Parkinson's Disease using Machine Learning Techniques", Kamal Narayan Reddy Challa* ,2016, IEEE

[2] Adoption of Electronic Health Record System: Multiple Theoretical Perspective", Qiwei Gan, Qing Cao - 2014 IEEE

[3] "A Scalable mHealth System for Noncommunicable Disease Management”, G D Clifford* - 2014 IEEE

[4] "Predictive Medication and use of BigData", Avijit Goswami 2017 IEEE

[5] "Variation in Outcome in Tethered Cord Syndrome", Norulain Iqbal*, 2016, Asian Spine Journal

[6] "Resource Frequency Prediction in Healthcare: Machine Learning Approach” Daniel Vieira, 2016 IEEE

[7] National Patient Safety Association, "Safer care for acutely ill patients:Learning from serious accidents," Tech. Rep., 2007.

[8] National Institute for Clinical Excellence, "Recognition of and response

to acute illness in adults in hospital," Tech. Rep., 2007. 
[9] H. Gao, A. McDonnell, D. Harrison, S. Adam, K. Daly, L. Esmonde, D. Goldhill, G. Parry, A. Rashidian, C. Subbe, and S. Harvey, "Systematic review and evaluation of physiological track and trigger warning systems for identifying at-risk patients on the ward," Intensive Care Med., vol. 33, no. 4, pp. 667-679, 2007.

[10] L. Tarassenko, D. Clifton, M. Pinsky, M. Hravnak, J. Woods, and P.Watkinson, "Centile-based early warning scores derived from statistical distributions of vital signs," Resuscitation, vol. 82, no. 8, pp. 1013-1018, 2011.

[11] D. Prytherch, G. Smith, P. Schmidt, P. Featherstone, K. Stewart,D.Knight,and B. Higgins, "Calculating early warning scores-A classroom comparisonof pen and paper and hand-held computer methods," Resuscitation,vol. 70, pp. 173-178, 2006.

[12] A. Hann, "Multi-parameter monitoring for early warning of patient deterioration,"Ph.D. dissertation, Univ. Oxford, Oxford, U.K., 2008.

[13] D. Wong, I. Strachan, and L. Tarassenko, "Visualisation of highdimensional data for very large data sets," presented at the Workshop Mach. Learn. Healthcare Appl., Helsinki, Finland, 2008.

[14] B. Schölkopf, J. Platt, J. Shawe-Taylor,A. J. Smola, and R C. Williamson, "Estimating the support of a high-dimensional distribution," Neural Comput., vol. 13, no. 7, pp. 1443-1471, 2001.

[15] S. Hugueny, D. Clifton, and L. Tarassenko, "Probabilistic patient monitoring with multivariate, multimodal extreme value theory," Commun. Comput. Sci., vol. 127, pp. 199-211, 2011.

[16] R. Kavitha, E. Kannan, S. Kotteswaran,"Implementation of Cloud based Electronic Health Record (EHR) for Indian Healthcare
Needs",Indian Journal of Science and Technology,2016 Jan, 9(3), Doi :10.17485/ijst/2016/v9i3/86391

[17] Meenakshi Sharma, Himanshu Aggarwal,"EHR Adoption in India: Potential and the Challenges",Indian Journal of Science and Technology, 2016 Sep, 9(34), Doi:10.17485/ijst/2016/v9i34/100211

[18] ResScan Software : ResScan version 4.2 Clinical Guide from "ResMed Ltd 1 Elizabeth Macarthur Drive Bella Vista NSW 2153 Australia"

[19] R. Lozano, and C. J. L. Murray, "Global and regional mortality from 235 causes of death for 20 age groups in 1990 and 2010: A systematic analysis for the Global Burden of Disease Study 2010," The Lancet, vol. 380, no. 9859, pp. 2095-2128, 2012.

[20] B. A. Payne, J. A. Hutcheon, "Arisk prediction model for the assessment and triage of women with hypertensivedisorders of pregnancy in low-resourced settings: The miniPIERS (Pre-eclampsia Integrated Estimate of RiSk) multi-country prospective cohort study," PLoS Med., vol. 11, no. 1, e1001589, pp. 1-13, 2013.

[21] W. Karlen, G. A. Dumont, C. Petersen, J. Gow, J. Lim, J. Sleiman, and J. M. Ansermino, "Human-centered phone oximeter interface design for the operating room," in Proc. Inter. Conf. Health form., Rome, Italy, 2011, pp. 433-438.

[22] University of British Columbia. (2014) Community level interventions for pre-eclampsia (CLIP). [Online]. Available: http://clinicaltrials.gov/ct2/show/NCT01911494. NLM Identifier: NCT01911494 